Vlado A. Lubarda

\title{
Radial stretching of a thin hollow membrane: biaxial tension, tension field and buckling domains
}

Received: 25 September 2010 / Published online: 17 November 2010

(C) The Author(s) 2010. This article is published with open access at Springerlink.com

\begin{abstract}
The radial stretching of a hollow thin membrane without compressive strength is considered within the framework of the small strain tension field theory. For each type of the uniform boundary conditions, the loading plane is partitioned into the domains of biaxial tension, tension field and buckling. The extent of these domains critically depends on the value of the Poisson's coefficient and on the aspect ratio of the membrane. The stress and displacement fields are determined at an arbitrary stage of loading, when the outer biaxially stressed (taut) annulus surrounds the inner (tension field) portion of the membrane, characterized by continuously distributed infinitesimal wrinkles. The growth of the tension field as the loading increases is analyzed. It is shown that, depending on the Poisson's coefficient and the aspect ratio of the membrane, the tension field may or may not spread throughout the whole membrane. For the fixed outer boundary, and the applied tension or a negative displacement at the inner boundary, and for a particular combination of the material and geometric parameters, the tension field instantly spreads to a specific depth within the membrane, dependent on the Poisson's ratio and the outer radius of the membrane, remaining constant during further increase of the loading.
\end{abstract}

\section{Introduction}

The stress and deformation analysis of thin membranes is a classical topic of interest for studying the mechanical response of metallic, semiconductor, and polymeric thin films and foils, textile panels, and biological membranes, such as skin or cell membranes [1-6]. If a thin membrane is assumed to lack compressive strength, its mechanical response is commonly analyzed by the tension field theory, originated in the early work of Wagner [7] and Reisner [8], and further developed by Kondo et al. [9], Stein and Hedgepeth [10], Pipkin [11], and Steigmann [12]. In this paper, we present a complete solution of the problem of radial stretching of a hollow circular membrane within the small strain tension field theory, extending and generalizing the earlier work devoted to this problem. The tension boundary, above which no compressive stress appears in the membrane, and the limit boundary, below which the membrane cannot support further loading without unstable buckling, are derived for each of the four types of loading conditions at the inner and outer boundary (stress, displacement, and two types of mixed boundary conditions). For loadings between the tension and limit boundaries, continuously distributed infinitesimal wrinkles appear within the inner portion of the membrane, carrying radial

\footnotetext{
V. A. Lubarda $(\varangle)$

Department of Mechanical and Aerospace Engineering, University of California,

San Diego, La Jolla, CA 92093-0411, USA

E-mail: vlubarda@ucsd.edu

Tel.: +1-858-5343169

Fax: $+1-858-5345698$

V. A. Lubarda

Montenegrin Academy of Sciences and Arts, Rista Stijovića 5, 81000 Podgorica, Montenegro
} 
tension, but no circumferential stress. The loading plane is partitioned into the domains of biaxial tension, uniaxial tension field, and buckling. The extent of these domains critically depends on the value of the Poisson's coefficient and on the aspect (radii) ratio of the membrane. The stress and displacement fields are determined at an arbitrary stage of loading, at which the outer biaxially stressed (taut) annulus surrounds the inner (tension field) portion of the membrane. The growth of the tension field with further increase of the loading is studied. It is shown that depending on the Poisson's coefficient and the aspect ratio of the membrane, the tension field may or may not spread throughout the whole membrane. The limiting extent of the tension field is determined. For the fixed outer boundary and the applied tension, or a negative displacement at the inner boundary, and for a particular combination of the material and geometric parameters, the tension field instantly spreads to a specific radius, dependent only on the Poisson's ratio and the outer radius of the membrane. This extent of the tension field remains constant during further increase of the loading.

The earlier work on the problem studied in this paper includes contributions [13-16], which were mainly in the context of the prescribed displacement boundary conditions, and did not address the partitioning of the full displacement range into biaxial tension, tension field, and buckling domains. Further referral to this work will be made in the body of this paper. A related research, using different constitutive models and finite strains, was conducted in [17,18] for rubber-like membranes, [19] for inelastic wrinkling of aluminum foils, [20-22] for human skin and soft tissues, and [23-25] for a viscoelastic and nonlinearly elastic erythrocyte membrane. One of the main objectives in these studies was to address the onset and propagation of membrane wrinkling. For example, in biomechanics, when a wound heals, it contracts and wrinkles the neighboring skin [26]; in cell locomotion, the crawling cell causes wrinkling of the membrane substrate [27]; in the micropipette aspiration test of a red blood cell, a relatively flat portion of the cell near the mouth of the pipette may wrinkle [28], etc. Other, more involved membrane problems, regarding both the geometry and loading, have also been studied in the framework of the tension field theory [29-32].

\section{Basic equations}

If the thickness of the membrane is exceedingly small (e.g., an erythrocyte membrane), the model of continuum mechanics applies within the plane of the membrane only, and the inplane stresses are defined by the force/length ratios $[33,34]$. Otherwise, a plane stress approximation is adopted within the usual threedimensional continuum analysis. In the former case, the coefficient of lateral contraction of an isotropic membrane is in the range $[0,1]$, and in the latter case $[0,1 / 2]$, where the value $v=1$ corresponds to isoareal membranes, and the value $v=1 / 2$ to incompressible membranes, respectively. The analysis presented in this paper encompasses both types of membranes (exceedingly thin, and thin), with the understanding that different definitions of stress and different bounds on the coefficient of lateral contraction apply in the two cases.

Consider a thin hollow circular membrane under uniform (traction, displacement, or mixed) boundary conditions at the inner and outer boundary. The equilibrium equation relating the two nonvanishing stress components in polar coordinates is $\sigma_{r}-\sigma_{\theta}+r \mathrm{~d} \sigma_{r} / \mathrm{d} r=0$. The compatibility condition for infinitesimal elastic strain, in terms of stresses reads $\sigma_{r}-\sigma_{\theta}-r \mathrm{~d} \sigma_{\theta} / \mathrm{d} r=0$. The well-known solution of these equations is

$$
\sigma_{r}=p_{0}-\frac{C}{r^{2}}, \quad \sigma_{\theta}=p_{0}+\frac{C}{r^{2}},
$$

where $p_{0}$ and $C$ are the constants to be determined from the boundary conditions. The radial and circumferential strains are defined in terms of the radial displacement $u=u(r)$ by $\epsilon_{r}=\mathrm{d} u / \mathrm{d} r$ and $\epsilon_{\theta}=u / r$, and are related by the strain compatibility condition $\epsilon_{r}=\mathrm{d}\left(r \epsilon_{\theta}\right) / \mathrm{d} r$. The constitutive expressions are

$$
\sigma_{r}-\sigma_{\theta}=\frac{E}{1+v}\left(\epsilon_{r}-\epsilon_{\theta}\right), \quad \sigma_{r}+\sigma_{\theta}=\frac{E}{1-v}\left(\epsilon_{r}+\epsilon_{\theta}\right),
$$

where $E$ is the inplane modulus of elasticity of the membrane. The displacement is $u=r \epsilon_{\theta}=r\left(\sigma_{\theta}-v \sigma_{r}\right) / E$, which gives

$$
u=\frac{1}{E}\left[(1-v) p_{0} r+(1+v) \frac{C}{r}\right]
$$

Since $\sigma_{r}+\sigma_{\theta}=2 p_{0}=$ const., and $\epsilon_{z}=-v\left(\sigma_{r}+\sigma_{\theta}\right) / E$, the change of the membrane thickness is uniform throughout the membrane. 


\section{Stress boundary conditions: prescribed $(p, q)$}

If the inner and outer boundaries of the membrane ( $r=a$ and $r=b)$ are subjected to radial tensions $p$ and $q$, i.e., $\sigma_{r}(a)=p$ and $\sigma_{r}(b)=q$, from (1) it readily follows that

$$
p_{0}=\frac{b^{2} q-a^{2} p}{b^{2}-a^{2}}, \quad C=\frac{a^{2} b^{2}}{b^{2}-a^{2}}(q-p) .
$$

Thus, the stresses before the onset of wrinkling are given by the well-known Lamé expressions (e.g., [35])

$$
\sigma_{r}=\frac{b^{2} q-a^{2} p}{b^{2}-a^{2}}-\frac{a^{2} b^{2}}{b^{2}-a^{2}} \frac{q-p}{r^{2}}, \quad \sigma_{\theta}=\frac{b^{2} q-a^{2} p}{b^{2}-a^{2}}+\frac{a^{2} b^{2}}{b^{2}-a^{2}} \frac{q-p}{r^{2}} .
$$

The corresponding radial displacement is

$$
u(r)=\frac{1}{E\left(b^{2}-a^{2}\right)}\left[(1-v)\left(b^{2} q-a^{2} p\right) r+(1+v) a^{2} b^{2} \frac{q-p}{r}\right] .
$$

\subsection{Tension field}

If $q \geq p$, both radial and circumferential stresses in (5) are positive throughout the membrane. If $q<p$, then $\sigma_{\theta}(r)$ is a an increasing function of $r$, and in order that tension prevails throughout the membrane, it is sufficient to require $\sigma_{\theta}(a) \geq 0$. The tension boundary in the $(p, q)$ loading plane, defined by $\sigma_{\theta}(a)=0$, is thus a straight line

$$
q=\frac{1}{2}\left(1+\frac{a^{2}}{b^{2}}\right) p
$$

If the loading state lies below the tension boundary, there is a tension field within $a \leq r \leq \rho$, where $r=\rho$ is the radius at which the hoop stress vanishes, $\sigma_{\theta}(\rho)=0$ (Fig. 1a). Within the tension field region, the constitutive expressions are $\sigma_{r}=E_{r} \epsilon_{r}$ and $\sigma_{\theta}=0$. The modulus of elasticity in the circumferential direction is zero $\left(E_{\theta}=0\right)$, because the membrane is assumed to be infinitesimally wrinkled in that direction. The modulus of elasticity in the radial direction is assumed to be constant and equal to its value $E_{r}=E$ at the boundary $r=\rho$. Consequently,

$$
\sigma_{r}=E \frac{\mathrm{d} u}{\mathrm{~d} r}
$$

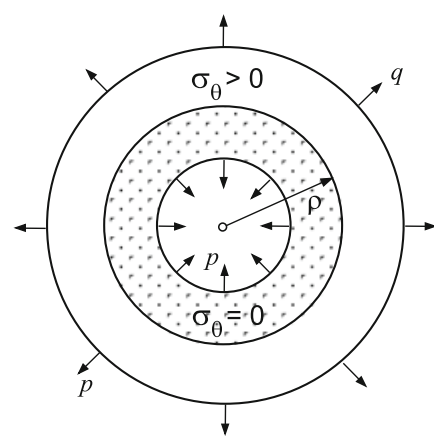

(a)

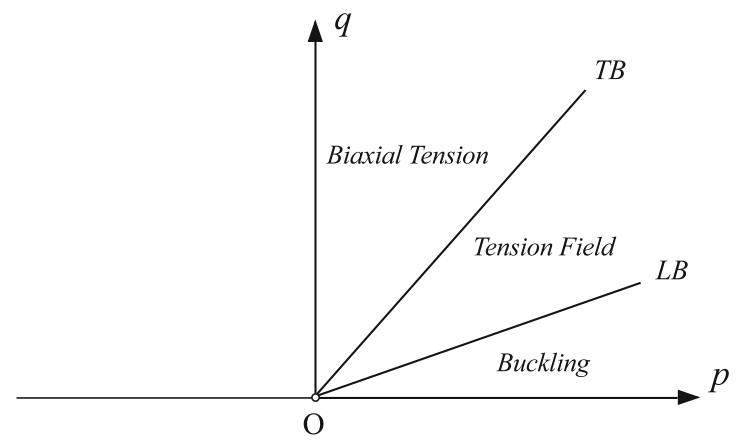

(b)

Fig. 1 a Within the tension field $(a \leq r \leq \rho)$, the membrane supports only the radial stress, while the circumferential stress is identically equal to zero $\left(\sigma_{\theta}=0\right)$. Outside the tension field $(\rho<r \leq b)$, both radial and circumferential tensile stresses act. The inner and outer radii of the membrane are $a$ and $b$. b The partitioning of the $(p, q)$ loading plane into three indicated domains (biaxial tension, tension field, and buckling) by the tension boundary $q=\left(1+a^{2} / b^{2}\right) p / 2$ and the limit boundary $q=(a / b) p$ 
The equation of equilibrium in the wrinkled region is $r \mathrm{~d} \sigma_{r} / \mathrm{d} r+\sigma_{r}=0$, which has the solution

$$
\sigma_{r}=p \frac{a}{r}=t \frac{\rho}{r} .
$$

The radial stress at $r=\rho$ is denoted by $t=\sigma_{r}(\rho)$. By equating (8) and (9), there follows:

$$
E \frac{\mathrm{d} u}{\mathrm{~d} r}=p \frac{a}{r}, \quad a \leq r \leq \rho .
$$

The boundary condition for this differential equations can be derived as follows. From Hooke's law, the radial stress at $r=\rho$ is $t=-E \epsilon_{\theta}(\rho) / \nu$, because $\sigma_{\theta}(\rho)=0$. Since $\epsilon_{\theta}(\rho)=u_{\rho} / \rho$ and since, from (9), $t=p a / \rho$, we obtain $u_{\rho}=-v p a / E$. Consequently, the integration of (10) gives

$$
u=-\frac{p a}{E}\left(\ln \frac{\rho}{r}+v\right), \quad a \leq r \leq \rho .
$$

The minus sign indicates that the displacement within the tension field is necessarily directed inward.

\subsection{Effective properties within the tension field}

The radial and hoop strains in the wrinkled region are

$$
\epsilon_{r}=\frac{\mathrm{d} u}{\mathrm{~d} r}=\frac{p}{E} \frac{a}{r}, \quad \epsilon_{\theta}=\frac{u}{r}=-\frac{p}{E} \frac{a}{r}\left(\ln \frac{\rho}{r}+v\right) .
$$

The coefficient of lateral contraction in the circumferential direction due to radial stress is

$$
v_{r \theta}=-\frac{\epsilon_{\theta}}{\epsilon_{r}}=\ln \frac{\rho}{r}+v \geq v, \quad a \leq r \leq \rho .
$$

The effective areal strain is

$$
\epsilon_{r}+\epsilon_{\theta}=\left(1-v_{r \theta}\right) \epsilon_{r}=\left(1-v-\ln \frac{\rho}{r}\right) \frac{p a}{E r}, \quad a \leq r \leq \rho .
$$

The corresponding effective areal modulus is

$$
\kappa=\frac{1}{2} \frac{\sigma_{r}}{\epsilon_{r}+\epsilon_{\theta}}=\frac{E}{2\left(1-v_{r \theta}\right)}, \quad a \leq r \leq \rho .
$$

From (14), it follows that for $\ln (\rho / a)>1-v$ the areal strain is negative within the portion of the membrane $a<r<\rho \exp (v-1)$. This means that, due to wrinkling, the (projected, in-plane) area of the membrane locally decreases, in spite of being under tensile stress. For $v=1$, the areal strain and, thus, the areal modulus $\kappa$, are negative throughout the tension field.

The strain energy densities inside $\left(\phi_{*}\right)$ and outside $(\phi)$ of the tension field are

$$
\begin{aligned}
\phi_{*} & =\frac{1}{2} \sigma_{r} \epsilon_{r}=\frac{1}{2} E \epsilon_{r}^{2}=\frac{p^{2} a^{2}}{2 E} \frac{1}{r^{2}}, \quad a \leq r \leq \rho, \\
\phi & =\frac{1}{2}\left(\sigma_{r} \epsilon_{r}+\sigma_{\theta} \epsilon_{\theta}\right)=\frac{E}{2\left(1-v^{2}\right)}\left(\epsilon_{r}^{2}+\epsilon_{\theta}^{2}+2 v \epsilon_{r} \epsilon_{\theta}\right) \\
& =\frac{p^{2} a^{2}}{4 E}\left[(1-v) \frac{1}{\rho^{2}}+(1+v) \frac{\rho^{2}}{r^{4}}\right], \quad \rho \leq r \leq b .
\end{aligned}
$$

The total work (per unit thickness) on stretching the membrane is thus

$$
W=\frac{\pi p^{2} a^{2}}{E}\left\{v+\ln \frac{\rho}{a}+\frac{q b}{2 p a}\left[(1-v) \frac{b}{\rho}-(1+v) \frac{\rho}{b}\right]\right\} .
$$


3.3 Stresses and displacement outside the tension field

The outside fields can be derived by considering the membrane outside the tension field to be under internal tension $t=\sigma_{r}(\rho)$ and external tension $q$. Thus, by replacing in (5) $p$ with $t$, and $a$ with $\rho$, the stresses outside the wrinkled region are

$$
\sigma_{r}=\frac{b^{2} q-\rho^{2} t}{b^{2}-\rho^{2}}-\frac{\rho^{2} b^{2}}{b^{2}-\rho^{2}} \frac{q-t}{r^{2}}, \quad \sigma_{\theta}=\frac{b^{2} q-\rho^{2} t}{b^{2}-\rho^{2}}+\frac{\rho^{2} b^{2}}{b^{2}-\rho^{2}} \frac{q-t}{r^{2}} .
$$

Since $\sigma_{\theta}(\rho)=0$, Eq. (17.2) gives

$$
t=\frac{2 b^{2} q}{\rho^{2}+b^{2}}
$$

which is also equal to $p a / \rho$, by (9). By substituting (18) back into (17), the stresses become

$$
\sigma_{r}=\frac{b^{2} q}{b^{2}+\rho^{2}}\left(1+\frac{\rho^{2}}{r^{2}}\right), \quad \sigma_{\theta}=\frac{b^{2} q}{b^{2}+\rho^{2}}\left(1-\frac{\rho^{2}}{r^{2}}\right)
$$

or

$$
\sigma_{r}=\frac{p a}{2 \rho}\left(1+\frac{\rho^{2}}{r^{2}}\right), \quad \sigma_{\theta}=\frac{p a}{2 \rho}\left(1-\frac{\rho^{2}}{r^{2}}\right)
$$

Likewise, from (6), the corresponding displacement is

$$
u=\frac{q}{E} \frac{b^{2}}{b^{2}+\rho^{2}}\left[(1-v) r-(1+v) \frac{\rho^{2}}{r}\right], \quad \rho \leq r \leq b
$$

or

$$
u=\frac{p a}{2 E}\left[(1-v) \frac{r}{\rho}-(1+v) \frac{\rho}{r}\right], \quad \rho \leq r \leq b .
$$

\subsection{The extent of the tension field}

The remaining unknown in the analysis is the extent of the tension field $\rho$. To derive an expression for $\rho$, we equate the expression $t=p a / \rho$, following from (9), with the expression (18). This yields the quadratic equation

$$
\rho^{2}-\frac{2 b^{2} q}{a p} \rho+b^{2}=0
$$

Its relevant solution (satisfying the condition $\rho<b$ ) is

$$
\frac{\rho}{b}=\frac{b q}{a p}\left[1-\sqrt{1-\left(\frac{a p}{b q}\right)^{2}}\right]
$$

because $b q>a p$. Note that, for a given loading pair $(p, q)$ below the tension boundary, the extent of the tension field $\rho$ in (24) is independent of the elastic constants $E$ and $\nu$. Later in this paper, we show that for the mixed type boundary conditions, as well as for the displacement boundary conditions, $\rho$ does depend on the elastic constants, in addition to being dependent on $a / b$ and the membrane loading ratio. 


\subsection{Limit boundary}

The limit boundary follows from (24) by setting $\rho=b$, so that the tension field spreads throughout the whole membrane. The limit boundary is thus a straight line $q=p a / b$. The corresponding stress in the membrane at that instant is $\sigma_{r}=p a / r=q b / r$, while the displacement is given by (11) with $\rho=b$. For example, if $q=0$, the membrane cannot support any $p$ without buckling, because the positive $p$-axis is under the limit boundary. On the other hand, if $p=0$, the membrane can support any tension $q$ (before rapture or other tensile failure), because the positive $q$-axis is above the tension boundary (Fig. 1b). For a very small hole in a large membrane $(b \gg a$ ), the tension boundary approaches the line $q=p / 2$, while the limit boundary approaches the horizontal line $q=0$.

The work done to bring the membrane to the limit state is obtained from (16) by substituting $\rho=b$ and $q / p=a / b$, with the end result $W_{\mathrm{LB}}=\left(\pi p^{2} a^{2} / E\right) \ln (b / a)$, independently of the Poisson's ratio. The membrane cannot support further loading beyond the limit state, because of the onset of unstable buckling, the analysis of which would require the incorporation of small but finite compressive strength and the associated bending stiffness of the membrane $[11,31,36]$. The incorporation of the bending stiffness is also needed to determine the size and shape of small wrinkles developed in the tension region of the tension field theory $[5,18]$.

\section{Mixed boundary conditions: prescribed $\left(u_{a}, q\right)$}

If displacement is prescribed at the inner boundary of the membrane, and tension at the outer boundary, $u(a)=u_{a}$ and $\sigma_{r}(b)=q$, from (3) there follows

$$
\frac{C}{b^{2}}=\frac{E\left(u_{a} / a\right)-(1-v) q}{1-v+(1+v)(b / a)^{2}}, \quad p_{0}=q+C / b^{2} .
$$

The denominator of the expression for $C / b^{2}$ is always positive, because $0 \leq v \leq 1$. The stress components before the onset of wrinkling are, therefore,

$$
\sigma_{r}=q+\frac{C}{b^{2}}\left(1-\frac{b^{2}}{r^{2}}\right), \quad \sigma_{\theta}=q+\frac{C}{b^{2}}\left(1+\frac{b^{2}}{r^{2}}\right),
$$

and the corresponding displacement

$$
u=\frac{1}{E}\left\{(1-v) q r+\frac{C}{b^{2}}\left[(1-v) r+(1+v) \frac{b^{2}}{r}\right]\right\} .
$$

\subsection{Tension field}

If $C \geq 0$ in (25), i.e., if $u_{a} / a \geq(1-v) q / E$, the hoop stress in (26) is necessarily positive, while $\mathrm{d} \sigma_{r} / \mathrm{d} r \geq 0$. Consequently, when $C \geq 0$, in order that the radial stress is non-negative throughout the membrane, it is sufficient to require $\sigma_{r}(a) \geq 0$, which implies

$$
(1-v) \frac{q}{E} \leq \frac{u_{a}}{a} \leq \frac{2}{1-a^{2} / b^{2}} \frac{q}{E} .
$$

If $u_{a} / a$ is larger than the right-hand side of the above inequality, the radial stress near the inner boundary becomes negative and the membrane will buckle.

If $C \leq 0$, i.e., $u_{a} / a \leq(1-v) q / E$, then $\sigma_{r}$ in (26) is necessarily positive, while $\mathrm{d} \sigma_{\theta} / \mathrm{d} r \geq 0$. Consequently, in this case, in order that the circumferential stress is non-negative throughout the membrane, it is sufficient to require $\sigma_{\theta}(a) \geq 0$, which implies

$$
-\frac{2 v}{1+a^{2} / b^{2}} \frac{q}{E} \leq \frac{u_{a}}{a} \leq(1-v) \frac{q}{E} .
$$

In particular, if $u_{a}=0$, the membrane is in a state of biaxial tension for any $q>0$. If $u_{a} / a$ is smaller than the left-hand side of the above inequality, the circumferential stress near the inner boundary becomes negative. 

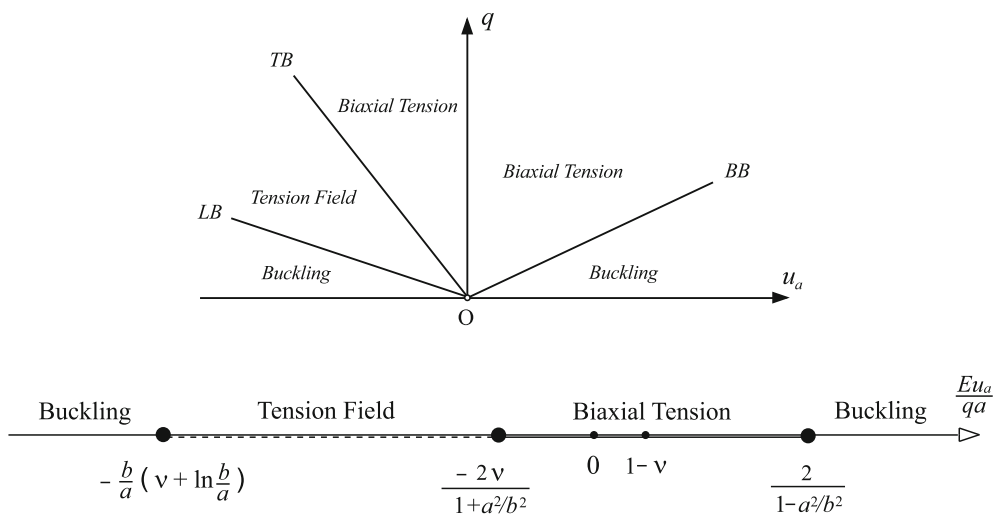

Fig. 2 The partitioning of the $\left(u_{a}, q\right)$ plane into biaxial tension, tension field, and buckling domains. The tension boundary $T B$ is given by (30), and the limit boundary $L B$ by (34). The buckling boundary $B B$ at positive $u_{a}$ is $q / E=\left(1-a^{2} / b^{2}\right) u_{a} / 2 a$. Also shown is the partitioning of the loading ratio $E u_{a} / q a$ range into the biaxial tension range $\left(\sigma_{r}>0, \sigma_{\theta}>0\right)$, tension field range $\left(\sigma_{r}>0, \sigma_{\theta}=0\right)$, and two buckling ranges: left from the tension field $\left(\sigma_{\theta}<0\right)$, and right from the biaxial tension $\left(\sigma_{r}<0\right)$

The membrane can support such displacements to some extent, by developing the tension field near the inner boundary (Fig. 2), carrying radial but no circumferential stress, as discussed in the sequel.

The tension boundary in the $\left(u_{a}, q\right)$ plane, defined by $\sigma_{\theta}(a)=0$, is a straight line

$$
\frac{q}{E}=-\frac{1}{2 v}\left(1+\frac{a^{2}}{b^{2}}\right) \frac{u_{a}}{a}
$$

with negative slope, inversely proportional to Poisson's ratio $v$ (Fig. 2). If $v=0$, the tension boundary is the vertical line $u_{a}=0$.

If the loading state is below the tension boundary, there is a tension field within $a \leq r \leq \rho$, where $\rho$ is defined by $\sigma_{\theta}(\rho)=0$. Within the tension field region $\sigma_{\theta}=0$, and $\sigma_{r}=E \mathrm{~d} u / \mathrm{d} r$, so that the equilibrium equation gives $\sigma_{r}=t \rho / r$. By equating this to $E \mathrm{~d} u / \mathrm{d} r$, we obtain a differential equation for $u$, whose solution is

$$
u=\frac{t \rho}{E} \ln \frac{r}{a}+u_{a}
$$

Thus, in view of the expression (18) for $t=\sigma_{r}(\rho)$, the stress and displacement within the tension field are

$$
\sigma_{r}=\frac{2 b^{2} q}{b^{2}+\rho^{2}} \frac{\rho}{r}, \quad u=\frac{2 q}{E} \frac{b^{2} \rho}{b^{2}+\rho^{2}} \ln \frac{r}{a}+u_{a}
$$

The stresses outside the wrinkled region are given by (19), and the displacement by (21), provided that $\rho$ is known.

\subsection{The extent of the tension field}

To determine the radius $\rho$, which specifies the extent of the tension field, the continuity of the displacement across $r=\rho$ is imposed. In view of (27) and (32), this gives

$$
\frac{2 q}{E} \frac{b^{2} \rho}{b^{2}+\rho^{2}}\left(v+\ln \frac{\rho}{a}\right)+u_{a}=0,
$$

which represents a nonlinear equation for $\rho$, to be solved numerically for each loading pair $\left(u_{a}, q\right)$ below the tension boundary. The plots of $\rho / a$ vs. the non-dimensional loading ratio $q a / E u_{a}$ are shown in Fig. 3, for the four selected values of Poisson's ratio $v$. 


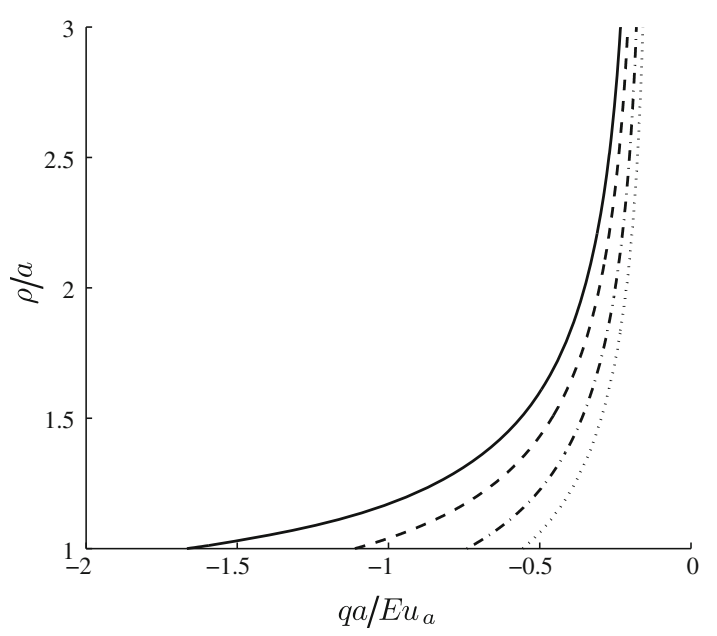

Fig. 3 The variation of $\rho / a$ with the loading ratio $q a / E u_{a}$ in the case $b=3 a$. The solid curve is for $v=1 / 3$, the dashed curve for $v=1 / 2$, the dotted-dashed for $v=3 / 4$, and the dotted for $v=1$. The end points of the curves correspond to the tension boundary $(\rho=a)$, and the limit boundary $(\rho=b)$ states of the membrane

\subsection{Limit boundary}

The limit boundary is obtained from (33) by setting $\rho=b$. This gives

$$
\frac{q}{E}=-\frac{a / b}{v+\ln (b / a)} \frac{u_{a}}{a}
$$

The tension $p$ at that instant is $p=q b / a$, while $u_{b}=-v q / E$. The expression (34) is used in Fig. 2 to complete the partitioning of the loading ratio range $E u_{a} / q a$ into the biaxial tension range $\left(\sigma_{r}>0, \sigma_{\theta}>0\right)$, the tension field range $\left(\sigma_{r}>0, \sigma_{\theta}=0\right)$, and two unstable buckling ranges $\left(\sigma_{r}<0\right)$ and $\left(\sigma_{\theta}<0\right)$. Thus, while in the case of the prescribed stresses $(p$ and $q$ ) the slopes of the tension and limit boundaries do not depend on the Poisson's ratio, in the case of the mixed boundary conditions the slopes are specified by the parameter that depends on the Poisson's ratio and the membrane aspect ratio $a / b$. The same is true for the other type of mixed boundary conditions $\left(p, u_{b}\right)$, considered in Sect. 5 , and the displacement boundary conditions $\left(u_{a}, u_{b}\right)$, considered in Sect. 6.

\section{Mixed boundary conditions: prescribed $\left(p, u_{b}\right)$}

If tension is prescribed at the inner boundary of the membrane, and displacement at the outer boundary, $\sigma_{r}(a)=p$ and $u(b)=u_{b}$, from (3) there follows

$$
\frac{C}{a^{2}}=\frac{E\left(u_{b} / b\right)-(1-v) p}{1-v+(1+v)(a / b)^{2}}, \quad p_{0}=p+C / a^{2} .
$$

The denominator of the first expression is always positive, because $0 \leq v \leq 1$. The stress components before the onset of wrinkling are, therefore,

$$
\sigma_{r}=p+\frac{C}{a^{2}}\left(1-\frac{a^{2}}{r^{2}}\right), \quad \sigma_{\theta}=p+\frac{C}{a^{2}}\left(1+\frac{a^{2}}{r^{2}}\right),
$$

with the corresponding displacement

$$
u=\frac{1}{E}\left\{(1-v) p r+\frac{C}{a^{2}}\left[(1-v) r+(1+v) \frac{a^{2}}{r}\right]\right\} .
$$


If $C \geq 0$, i.e., if $u_{b} / b \geq(1-v) p / E$, then $\sigma_{r}$ and $\sigma_{\theta}$ in (36) are both positive. If $C<0$, i.e., $u_{b} / b<(1-v) p / E$, the radial stress is monotonically decreasing, and the circumferential stress is a monotonically increasing function of $r$. Thus, for the positive-definiteness of the state of stress throughout the membrane, it is sufficient to require $\sigma_{r}(b)>0$ and $\sigma_{\theta}(a)>0$. The condition $\sigma_{r}(b)>0$ gives

$$
\frac{u_{b}}{b}>-2 \frac{a^{2} / b^{2}}{1-a^{2} / b^{2}} \frac{p}{E}
$$

This condition is certainly satisfied when $u_{b} \geq 0$, but has to be verified for $u_{b}<0$. In the range of negative $u_{b}$ for which (38) does not hold, the membrane will buckle, because $\sigma_{r}(b)$ becomes compressive. The condition $\sigma_{\theta}(a)>0$ gives

$$
\frac{u_{b}}{b}>\frac{1}{2}\left[1-v-(1+v) \frac{a^{2}}{b^{2}}\right] \frac{p}{E} .
$$

If this condition is not satisfied, the tension field may develop in the membrane, as discussed in the sequel. For later use, if $(1-v)<(1+v) a^{2} / b^{2}$, we note that

$$
2 \frac{a^{2} / b^{2}}{1-a^{2} / b^{2}}>\frac{1}{2}\left[(1+v) \frac{a^{2}}{b^{2}}-(1-v)\right],
$$

because $v \leq 1$.

\subsection{Tension field}

If there is a tension field developed in the range $a \leq r \leq \rho$, then

$$
\sigma_{r}=p \frac{a}{r}=t \frac{\rho}{r} .
$$

By equating this to $\sigma_{r}=E \mathrm{~d} u / \mathrm{d} r$, we obtain a differential equation for $u$, whose solution is

$$
u=\frac{p a}{E} \ln \frac{r}{\rho}+u_{\rho}=-\frac{p a}{E}\left(v+\ln \frac{\rho}{r}\right), \quad u_{\rho}=-\frac{v}{E} p a .
$$

The displacement $u_{\rho}$ is found from the expression $\epsilon_{\theta}(\rho)=-(\nu / E) t=u_{\rho} / \rho$, and $t=p a / \rho$. Note that $u_{\rho}=-v p a / E$ is also equal to $u_{a}$ at the onset of tension field $(\rho=a)$. Thus, interestingly, for a given $p$ and varying $u_{b}$ (causing the growth of the tension field), the interface displacement $u_{\rho}$ remains constant and equal to $u_{\rho}=-v p a / E$.

The stresses and displacement outside the tension field can be obtained from (35)-(37) by replacing $a$ with $\rho$, and $p$ with $t=p a / \rho$. This gives

$$
\sigma_{r}=p \frac{a}{\rho}+\frac{C_{0}}{\rho^{2}}\left(1-\frac{\rho^{2}}{r^{2}}\right), \quad \sigma_{\theta}=p \frac{a}{\rho}+\frac{C_{0}}{\rho^{2}}\left(1+\frac{\rho^{2}}{r^{2}}\right)
$$

where

$$
\frac{C_{0}}{\rho^{2}}=\frac{E\left(u_{b} / b\right)-(1-v) p a / \rho}{1-v+(1+v)(\rho / b)^{2}} .
$$

Since $\sigma_{\theta}(\rho)=0$, we have, from Eq. (43.2), $C_{0}=-p a \rho / 2$, so that (43) can also be cast as in (20). The corresponding displacement is given by (22). 

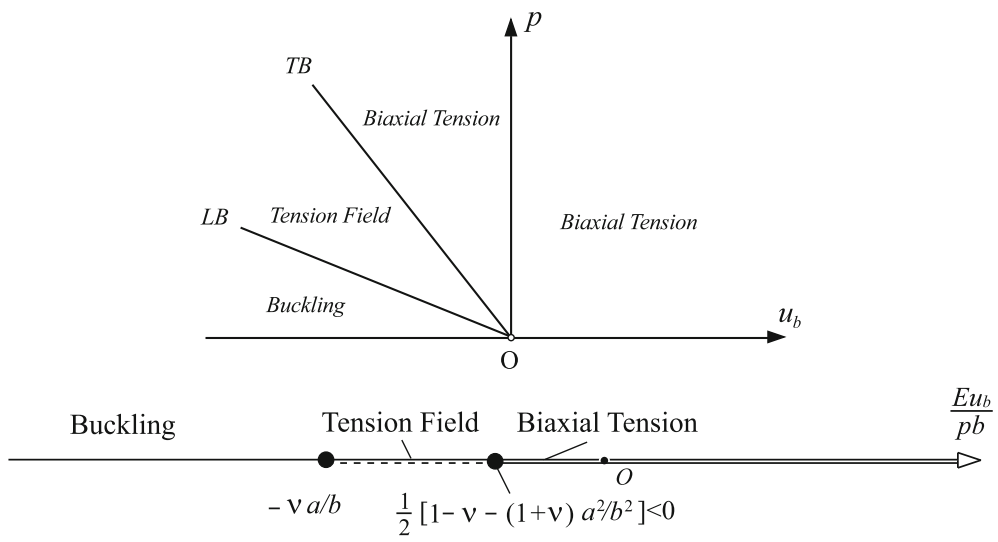

Fig. 4 The partitioning of the $\left(u_{b}, p\right)$ plane into biaxial tension $\left(\sigma_{r}>0, \sigma_{\theta}=0\right)$, tension field $\left(\sigma_{r}>0, \sigma_{\theta}>0\right)$, and buckling $\left(\sigma_{\theta}<0\right)$ domains, in the case $1-v-(1+v) a^{2} / b^{2}<0$. The tension boundary $T B$ is given by (48), and the limit boundary $L B$ by (51). Also shown is the partitioning of the loading ratio range $E u_{b} / p b$

\subsection{The extent of the tension field}

To determine the radius $\rho$, we impose the displacement continuity at $r=\rho$ (or require in (20) that $u(b)=u_{b}$, or equate (44) with $C_{0}=-p a \rho / 2$ ). Either of these gives

$$
2 E \frac{u_{b}}{b} \frac{\rho}{a}-p\left[(1-v)-(1+v)\left(\frac{\rho}{b}\right)^{2}\right]=0 .
$$

Four different cases need to be distinguished.

Case $1 \quad u_{b} \leq 0$ and $1-v-(1+v) a^{2} / b^{2}<0$

If

$$
\frac{u_{b}}{b}>\frac{1}{2}\left[1-v-(1+v) \frac{a^{2}}{b^{2}}\right] \frac{p}{E},
$$

the biaxial tension prevails throughout the membrane, and the tension field does not develop. If

$$
\frac{u_{b}}{b} \leq \frac{1}{2}\left[1-v-(1+v) \frac{a^{2}}{b^{2}}\right] \frac{p}{E},
$$

the tension field does set up (Fig. 4). The tension boundary is defined by $\sigma_{\theta}(a)=0$ and is a straight line

$$
\frac{u_{b}}{b}=\frac{1}{2}\left[1-v-(1+v) \frac{a^{2}}{b^{2}}\right] \frac{p}{E} .
$$

If $u_{b}$ is kept fixed, and $p$ decreases below the tension boundary, the tension field spreads from $r=a$ to $r=\rho$. The radius $\rho$ can be determined by solving the quadratic Eq. (45), i.e.,

$$
(1+v)\left(\frac{\rho}{b}\right)^{2}+2 \frac{E u_{b}}{p a}\left(\frac{\rho}{b}\right)-(1-v)=0
$$

whose solution is

$$
\frac{\rho}{b}=-\frac{1}{1+v} \frac{E u_{b}}{p a}\left\{1+\left[1+\left(1-v^{2}\right)\left(\frac{p a}{E u_{b}}\right)^{2}\right]^{1 / 2}\right\} .
$$

The limit boundary is obtained from (50) by setting $\rho=b$, which gives

$$
\frac{u_{b}}{a}=-v \frac{p}{E} \text {. }
$$



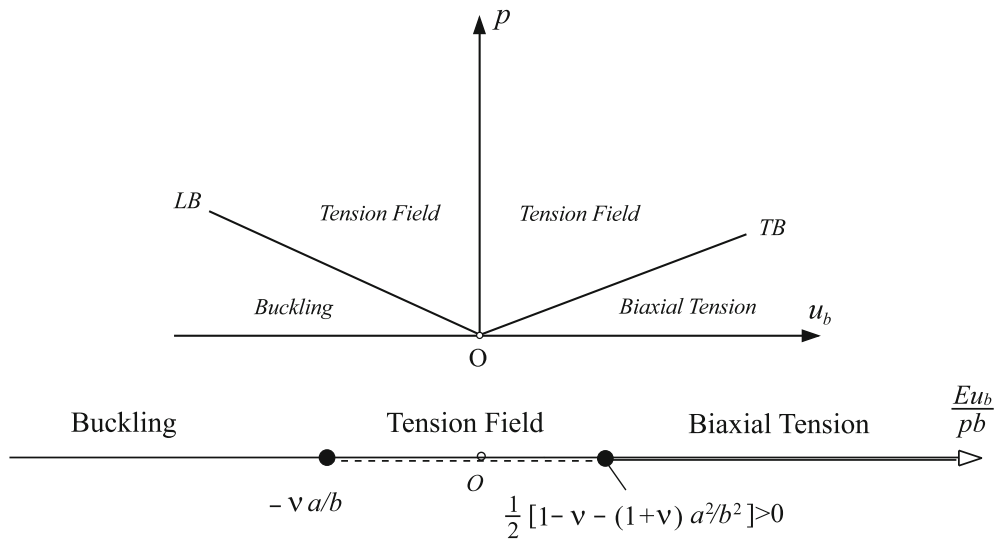

Fig. 5 The partitioning of the $\left(u_{b}, p\right)$ plane into biaxial tension, tension field, and buckling domains, in the case $1-v-(1+v)$ $a^{2} / b^{2}>0$. Also shown is the corresponding partitioning of the loading ratio range $E u_{b} /(p b)$

The tension $q$ at that instant is $q=(a / b) p / 2$. The expression (51) is used in Fig. 4 to complete the partitioning of the loading ratio range $E u_{b} /(p a)$ into the biaxial tension range $\left(\sigma_{r}>0, \sigma_{\theta}>0\right)$, the tension field range $\left(\sigma_{r}>0, \sigma_{\theta}=0\right)$, and the buckling range $\left(\sigma_{\theta}<0\right)$.

Case $2 u_{b}<0$ and $1-v-(1+v) a^{2} / b^{2}>0$

In this case, for any given $u_{b}<0$, the buckling takes place in the $p$-range from zero up to the $p$-value at the limit boundary state $(\rho=b)$, i.e.,

$$
0 \leq p \leq-\frac{E}{v} \frac{u_{b}}{a}
$$

As $p$ is further increased, the tension field shrinks from $\rho=b$ to the limiting value $\rho_{0}$, obtained by requiring in (45) that

$$
(1-v)-(1+v)\left(\frac{\rho}{b}\right)^{2} \rightarrow 0^{-}, \quad \text { as } \quad p \rightarrow \infty
$$

Thus,

$$
\rho_{0}=\left(\frac{1-v}{1+v}\right)^{1 / 2} b
$$

The tension $q$ at this limiting state scales with $p$ as $q / p=(a / b)\left(1-v^{2}\right)^{-1 / 2}$. Of course, if $p$ grows beyond some critical value, large strains and tensile rapture of the membrane would interrupt the limiting process $p \rightarrow \infty$. Figure 5 shows the buckling and the tension field domains in the $\left(u_{b}, p\right)$ plane, and along the $E u_{b} / p b$ axis.

Case $3 u_{b}>0$ and $1-v-(1+v) a^{2} / b^{2}>0$

If

$$
\frac{u_{b}}{b}>\frac{1}{2}\left[1-v-(1+v) \frac{a^{2}}{b^{2}}\right] \frac{p}{E},
$$

the biaxial tension prevails throughout the membrane, and the tension field does not develop. If

$$
\frac{u_{b}}{b} \leq \frac{1}{2}\left[1-v-(1+v) \frac{a^{2}}{b^{2}}\right] \frac{p}{E},
$$

the tension field sets up. The tension boundary is a straight line,

$$
\frac{u_{b}}{b}=\frac{1}{2}\left[1-v-(1+v) \frac{a^{2}}{b^{2}}\right] \frac{p}{E} .
$$




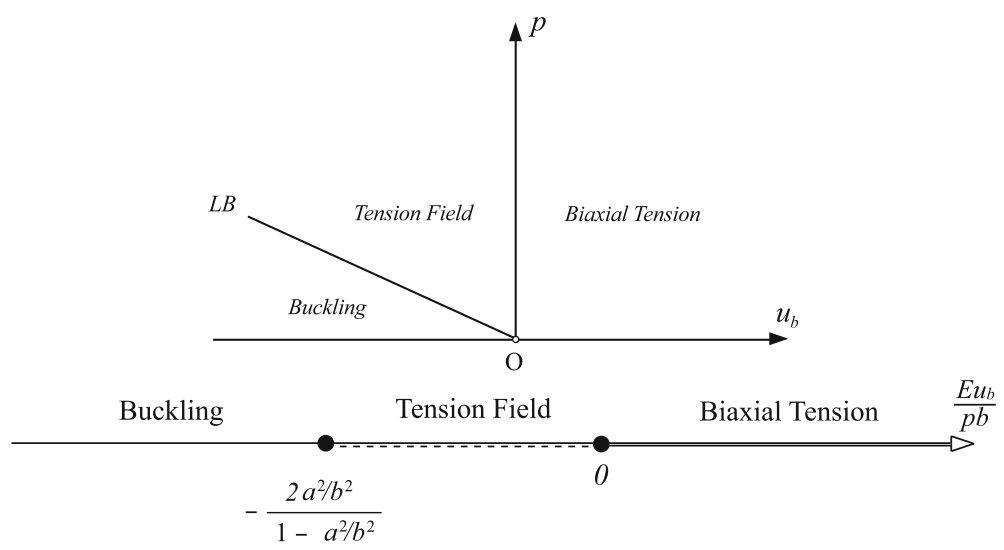

Fig. 6 The partitioning of the $\left(u_{b}, p\right)$ plane into biaxial tension, tension field, and buckling domains, in the case $1-v-(1+v)$ $a^{2} / b^{2}=0$. Also shown is the corresponding partitioning of the loading ratio range $E u_{b} / p b$

If $u_{b}$ is kept fixed, and $p$ increases above the tension boundary, the tension field spreads from $r=a$ to the limiting value (54), reached in the limit as $p \rightarrow \infty$. Thus, the maximum extent of the tension field is smaller for the membranes with larger Poisson's ratio (Poisson's ratio opposes wrinkling, as noted by Géminard et al. [5] and Coman [15]). In particular, if $v=0$, the tension field (infinitesimal wrinkling) extends throughout the whole membrane; if $v=1$, it does not extend at all. Figure 5 shows the biaxial and the tension field domains in the $\left(u_{b}, p\right)$ plane.

As $p$ increases above the tension boundary, the radius $\rho$ can be determined by solving the quadratic Eq. (45), whose relevant solution in this case is

$$
\frac{\rho}{b}=\frac{1}{1+v} \frac{E u_{b}}{p a}\left\{\left[1+\left(1-v^{2}\right)\left(\frac{p a}{E u_{b}}\right)^{2}\right]^{1 / 2}-1\right\} .
$$

Case $4 u_{b} \geq 0$ and $1-v-(1+v) a^{2} / b^{2}<0$

In this case,

$$
\frac{u_{b}}{b}>\frac{1}{2}\left[1-v-(1+v) \frac{a^{2}}{b^{2}}\right] \frac{p}{E},
$$

so that $\sigma_{\theta}(a)>0$, and biaxial tension prevails in the entire first quadrant of the $\left(u_{b}, p\right)$ plane, i.e., along the entire positive portion of the $E u_{b} / p b$ axis (Fig. 4).

Case $51-v-(1+v) a^{2} / b^{2}=0$

It readily follows that the tension boundary is $u_{b}=0$, so that biaxial tension prevails for all $u_{b} \geq 0$, at any value of positive $p$. Buckling occurs at negative $u_{b}$, if

$$
\frac{u_{b}}{b}<-2 \frac{a^{2} / b^{2}}{1-a^{2} / b^{2}} \frac{p}{E} .
$$

For $u_{b}$ in-between the tension boundary $u_{b}=0$ and the above buckling limit, the tension field develops, as depicted in Fig. 6.

\subsection{Special case: $u_{b}=0$}

If the outer boundary is fixed $\left(u_{b}=0\right)$, and if $(1-v) b / a-(1+v) a / b \leq 0$, biaxial tension prevails throughout the loading history $p>0$. On the other hand, if $u_{b}=0$ and $(1-v) b / a-(1+v) a / b>0$, the tension field instantly sets up in the range $a \leq r \leq \rho_{0}$ as soon as any, however small, positive tension $p$ is applied at the 
inner boundary. The extent of the tension field $\rho_{0}$ remains constant during further increase of $p$. The stress and displacement fields are, thus,

$$
\begin{aligned}
\sigma_{r} & =p \frac{a}{r}=t \frac{\rho_{0}}{r}, \quad a \leq r \leq \rho_{0}, \\
u & =-\frac{p a}{E}\left(v+\ln \frac{\rho_{0}}{r}\right), \quad \rho_{0}=\left(\frac{1-v}{1+v}\right)^{1 / 2},
\end{aligned}
$$

and

$$
\begin{aligned}
\sigma_{r} & =\frac{p a}{2 \rho_{0}}\left(1+\frac{\rho_{0}^{2}}{r^{2}}\right), \quad \sigma_{\theta}=\frac{p a}{2 \rho_{0}}\left(1-\frac{\rho_{0}^{2}}{r^{2}}\right) . \\
u & =\frac{p a}{2 E}\left[(1-v) \frac{r}{\rho_{0}}-(1+v) \frac{\rho_{0}}{r}\right], \quad \rho_{0} \leq r \leq b .
\end{aligned}
$$

\section{Displacement boundary conditions: prescribed $\left(u_{a}, u_{b}\right)$}

If the displacement boundary conditions are prescribed, $u(a)=u_{a}$ and $u(b)=u_{b}$, from (3) it follows that

$$
C=\frac{E a b}{1+v} \frac{b u_{a}-a u_{b}}{b^{2}-a^{2}}, \quad p_{0}=\frac{E}{1-v} \frac{b u_{b}-a u_{a}}{b^{2}-a^{2}} .
$$

The corresponding stresses and the displacement are given by the well-known expressions

$$
\begin{aligned}
\sigma_{r} & =\frac{E}{b^{2}-a^{2}}\left(\frac{b u_{b}-a u_{a}}{1-v}-\frac{b u_{a}-a u_{b}}{1+v} \frac{a b}{r^{2}}\right), \\
\sigma_{\theta} & =\frac{E}{b^{2}-a^{2}}\left(\frac{b u_{b}-a u_{a}}{1-v}+\frac{b u_{a}-a u_{b}}{1+v} \frac{a b}{r^{2}}\right), \\
u & =\frac{r}{b^{2}-a^{2}}\left[b u_{b}-a u_{a}+\left(b u_{a}-a u_{b}\right) \frac{a b}{r^{2}}\right] .
\end{aligned}
$$

We suppose in this section that $v<1$. Otherwise, $u_{a}$ and $u_{b}$ cannot be prescribed independently, because of the isoareal constraint $b u_{b}=a u_{a}$.

If $u_{a} \geq 0$, the membrane is in a state of biaxial tension $\left(\sigma_{r}>0\right.$ and $\left.\sigma_{\theta}>0\right)$, provided that

$$
u_{b}>\frac{1}{2}\left[(1-v) \frac{b}{a}+(1+v) \frac{a}{b}\right] u_{a} .
$$

If $u_{a} \geq 0$, and the inequality (67) is not satisfied, buckling occurs because $\sigma_{r}(a)$ becomes negative. If $u_{a} \leq$ 0 , and

$$
u_{b}<\frac{2 u_{a}}{(1-v)(a / b)+(1+v)(b / a)},
$$

buckling occurs, because $\sigma_{r}(b)$ becomes negative.

If $u_{a} \leq 0$, the biaxial tension prevails for

$$
u_{b}>\frac{1}{2 v}\left[(1+v) \frac{a}{b}-(1-v) \frac{b}{a}\right] u_{a}
$$

If $u_{a}<0$, and

$$
u_{b}<\frac{1}{2 v}\left[(1+v) \frac{a}{b}-(1-v) \frac{b}{a}\right] u_{a},
$$

the tension field develops, until the limit boundary is reached, below which buckling takes place. Figures 7 and 8 show the partitioning of the $\left(u_{a}, u_{b}\right)$ plane, which is discussed further in the next section. 


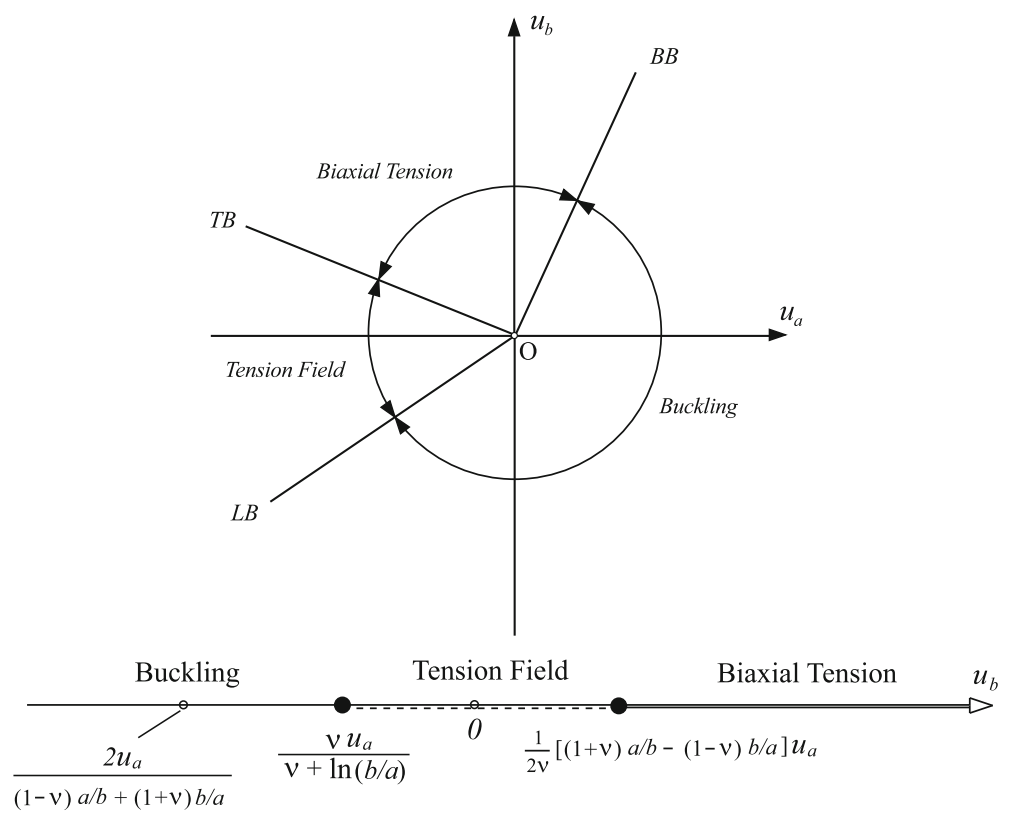

Fig. 7 The partitioning of the $\left(u_{a}, u_{b}\right)$ plane and the $u_{b}$ axis in the case $u_{a}<0$ and $(1+v) a / b-(1-v) b / a \leq 0$
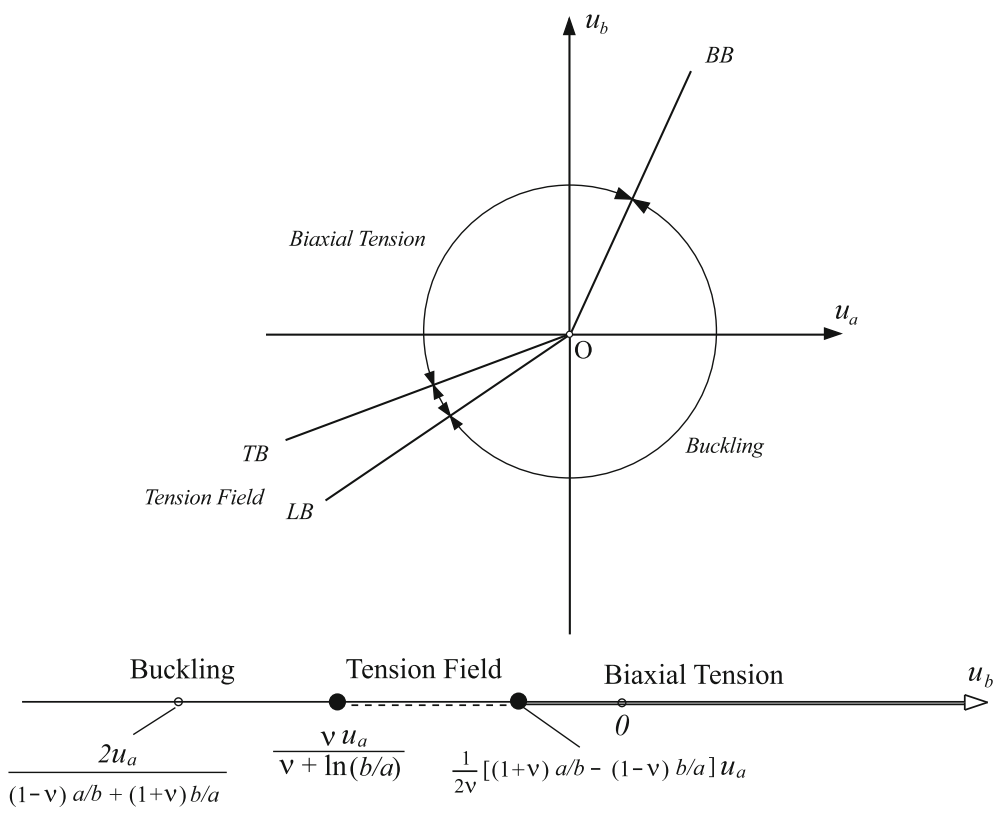

Fig. 8 The partitioning of the $\left(u_{a}, u_{b}\right)$ plane and the $u_{b}$ axis in the case $u_{a}<0$ and $(1+v) a / b-(1-v) b / a \geq 0$

\subsection{Tension field}

There is no tension field for $u_{a} \geq 0$ (Fig. 9), because the condition $\sigma_{\theta}(a)=0$ gives

$$
u_{b}=\frac{1}{2 v}\left[(1+v) \frac{a}{b}-(1-v) \frac{b}{a}\right] u_{a},
$$

which falls inside the buckling range

$$
u_{b}<\frac{1}{2}\left[(1-v) \frac{b}{a}+(1+v) \frac{a}{b}\right] u_{a},
$$




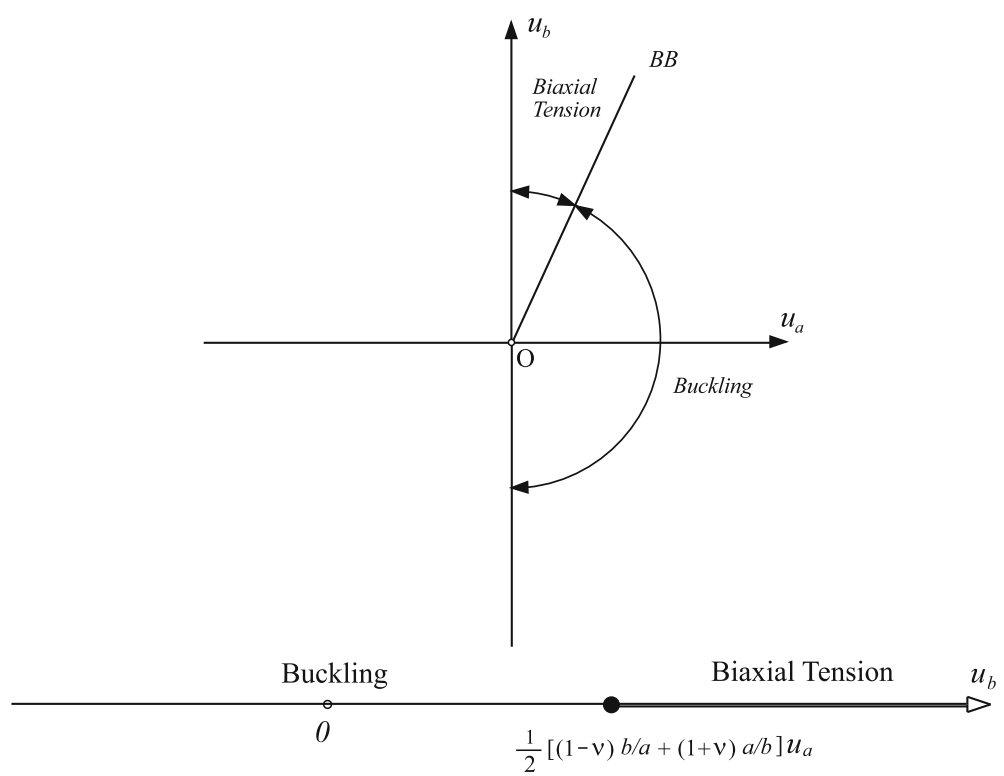

Fig. 9 The partitioning of the $\left(u_{a}, u_{b}\right)$ plane and the $u_{b}$ axis in the case $u_{a} \geq 0$. The straight line $B B$ is the biaxial tension/buckling boundary

associated with $\sigma_{r}(a)<0$. This is so because the coefficient multiplying $u_{a}$ in (72) is larger than the corresponding coefficient in (71). The absence of the tension field for $u_{a}>0$ is also clear from either (11) or (42), which (at $r=\rho=a$ ) give $u_{a}=-v p a / E$, or from (30), because the radial stresses at the inner and outer boundaries $(p$ and $q)$ must be positive.

For $u_{a}<0$, the tension field develops for the membrane states under the tension boundary

$$
u_{b}=\frac{1}{2 v}\left[(1+v) \frac{a}{b}-(1-v) \frac{b}{a}\right] u_{a} .
$$

If the tension field has spread to a radius $\rho$, the stress and displacement fields within and outside the tension field are given by (41) and (42), and (20) and (22). To determine $p$ and $\rho$, associated with the prescribed $u_{a}$ and $u_{b}$ below the tension boundary (73), we impose in these expressions the conditions $u(a)=u_{a}$ and $u(b)=u_{b}$. This gives

$$
\begin{gathered}
-\frac{p a}{E}\left(v+\ln \frac{\rho}{a}\right)=u_{a}, \\
\frac{p a}{2 E}\left[(1-v) \frac{b}{\rho}-(1+v) \frac{\rho}{b}\right]=u_{b} .
\end{gathered}
$$

Dividing (75) with (74) generates a nonlinear equation for $\rho$,

$$
(1-v) \frac{b}{\rho}-(1+v) \frac{\rho}{b}+2 \frac{u_{b}}{u_{a}}\left(v+\ln \frac{\rho}{a}\right)=0 .
$$

An equivalent equation was derived in [15], where the conditions under which this equation has a solution were examined. The plots of $\rho / a$ vs. $u_{b} / u_{a}$, in the case $b=3 a$ and for the three selected values of the Poisson's ratio, are shown in Fig. 10. For a given ratio $u_{b} / u_{a}$, the extent of the tension field is more pronounced for the membranes with lower value of the Poisson's ratio.

If the outer boundary is fixed $\left(u_{b}=0\right)$, the membrane will buckle for any applied $u_{a}>0$. If $u_{b}=0$ and $(1+v) a / b-(1-v) b / a \geq 0$, biaxial tension prevails throughout the loading history $u_{a}<0$. On the other hand, if $u_{b}=0$ and $(1+v) a / b-(1-v) b / a<0$, the tension field instantly sets up in the range $a \leq r \leq \rho_{0}$ as soon as any, however small, negative displacement $u_{a}<0$ is applied at the inner boundary. The extent of the tension field $\rho_{0}$ thereafter remains constant, as long as $u_{a}$ remains negative. Previously, Coman [15] observed a rapid increase in $\rho$ from $a$ to $\rho_{0}$, with the increase in the magnitude of $u_{a}<0$, at a very small constant value of the applied displacement $u_{b}>0$, but did not discuss the case $u_{b}=0$. Chiu et al. [37] considered the case 


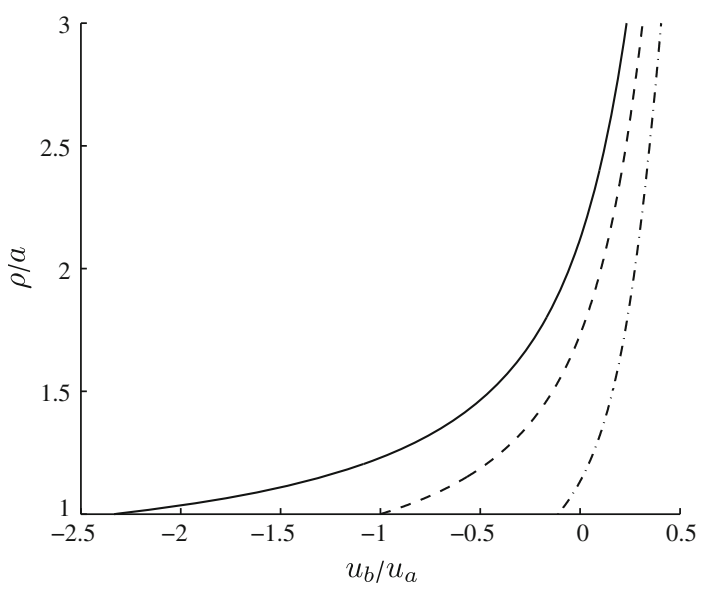

Fig. 10 The increase of the extent of the tension field with the increasing displacement ratio $u_{b} / u_{a}$, in the case $b=3 a$. The solid curve is for $v=1 / 3$, the dashed curve for $v=1 / 2$, and the dotted-dashed curve for $v=3 / 4$

$u_{b}=0$, but without observing the constraint $(1+v) a / b-(1-v) b / a<0$, required for the existence of the tension field between $r=a$ and $r=\rho_{0}$. Also, there is an error in their displacement expressions (37) and (38).

If $(1+v) a / b-(1-v) b / a<0$, and the displacement $u_{b}>0$ is held fixed, while $u_{a}<0$ increases in magnitude from zero to its value at the tension boundary state, and beyond into the tension field, $\rho$ increases from $a$ to its limiting value $\rho_{0}=[(1-v) /(1+v)]^{1 / 2} b$ (ignoring large strain effects and tensile rupture). Similarly, if $u_{b}<0$ is held fixed, while $u_{a}<0$ increases in magnitude beyond its value at the limit boundary state, into the tension field, $\rho$ decreases from $b$ to its limiting value $\rho_{0}$ (Fig. 7).

\subsection{Limit boundary}

The limit boundary is obtained from (76) by setting $\rho=b$. The resulting equation is

$$
\frac{u_{b}}{u_{a}}=\frac{v}{v+\ln (b / a)} .
$$

The partitioning of the $\left(u_{a}, u_{b}\right)$ plane and the axis $u_{b}$ into the biaxial tension, tension field, and the buckling domains is shown in Figs. 7 and 8 , depending on the value of the parameter $(1+v) a / b-(1-v) b / a$. The corresponding tensions $p$ and $q$, at the limit boundary state, are

$$
p=-\frac{E u_{a}}{a} \frac{1}{v+\ln (b / a)}, \quad q=-\frac{E u_{a}}{b} \frac{1}{v+\ln (b / a)},
$$

so that $q=(a / b) p$, in agreement with the results from Sects. 3, 4 and 5. It is noted that $v+\ln (b / a)$, appearing in (77) and (78), and earlier in (34), is equal to $v_{r \theta}(a)$ at the limiting state $\rho=b$; cf. (13).

\section{Conclusion}

We have presented in this paper a complete solution of the problem of radial stretching of a thin hollow membrane within the tension field theory. In the case of the prescribed tension $(p)$ at the inner boundary, there is a closed form solution for either the prescribed tension $(q)$ or prescribed displacement $\left(u_{b}\right)$ at the outer boundary. If the displacement $\left(u_{a}\right)$ is prescribed at the inner boundary, and the tension $(q)$ or the displacement $\left(u_{b}\right)$ at the outer boundary, the solution is in a closed form, apart for a nonlinear equation for the extent of the tension field, which needs to be solved numerically. In each of the four considered types of boundary conditions, the loading plane is divided into domains of biaxial tension, tension field, and buckling, as dictated by the corresponding interplay of the boundary conditions, and material and geometric parameters of the problem. It is shown that the extent of different domains critically depends on the Poisson's coefficient and the aspect (radii) ratio of the hollow membrane, except in the case of prescribed tensions at both boundaries, where the 
slopes of the tension and limit boundaries, which separate different domains, depend only on the aspect ratio of the membrane. In the case of prescribed displacement at the outer boundary, and either tension or displacement at the inner boundary, the tension field may or may not spread throughout the entire membrane, depending again on the values of the Poisson's coefficient and the aspect ratio of the membrane. If the outer boundary is fixed $\left(u_{b}=0\right)$, in certain cases it may happen that the tension field instantly spreads from the inner boundary to the radius $\rho_{0} \leq b$, as soon as either a negative displacement $u_{a}$ or positive tension $p$, however small, are applied at the inner boundary. The extent of this tension field thereafter remains constant, although $-u_{a}$ or $p$ further increase. The stress and displacement fields are determined in each considered case at an arbitrary stage of loading, when the outer taut annulus surrounds the inner infinitesimally wrinkled portion of the membrane.

Acknowledgment Research support from the Montenegrin Academy of Sciences and Arts is gratefully acknowledged. Technical help from the undergraduate students Elizabeth Zhao and Marina Gonzales in the preparation of the manuscript is also acknowledged.

Open Access This article is distributed under the terms of the Creative Commons Attribution Noncommercial License which permits any noncommercial use, distribution, and reproduction in any medium, provided the original author(s) and source are credited.

\section{References}

1. Mansfield, E.H.: Load transfer via a wrinkled membrane. Proc. R. Soc. Lond. A 316, 269-289 (1970)

2. $\mathrm{Wu}, \mathrm{C}$.-H.: Nonlinear wrinkling of nonlinear membranes of revolution. J. Appl. Mech. 45, 533-538 (1978)

3. Miller, R.K., Hedgepeth, J.M., Weingarten, V.I., Das, P., Kahyai, S.: Finite element analysis of partly wrinkled membranes. Comput. Struct. 20, 631-639 (1985)

4. Jenkins, C.H.: Nonlinear dynamic response of membranes: state of art—update. Appl. Mech. Rev. 49, S41-S48 (1996)

5. Géminard, J.-C., Berbal, R., Melo, F.: Wrinkle formations in axi-symmetrically stretched membranes. Eur. Phys. J. E 15, $117-126(2004)$

6. Agrawal, A., Steigmann, D.J.: Boundary-value problems in the theory of lipid membranes. Continuum Mech. Thermodyn. 21, 57-82 (2009)

7. Wagner, H.: Ebene Blechwandträger mit Sehr dünnem Stegblech. Zeitschr. Flugtechnik Motorluftschiffahrt 20, 8-12 (1929)

8. Reissner, E.: On tension field theory. In: Proceedings of Fifth International Congress of Applied Mechanics, pp. 88-92 (1938)

9. Kondo, K., Iai, T., Moriguti, S., Murasaki, T.: Tension-field theory. In: Kondo, K. (ed.) Memoirs of the Unifying Study of the Basic Problems in Engineering Sciences by Means of Geometry, Vol I, pp. 417-441. Gakujutsu Bunken Fukyu-Kai, Tokyo (1955)

10. Stein, M., Hedgepeth, J.M.: Analysis of partly wrinkled membranes. NASA TN D-813 (1961)

11. Pipkin, A.C.: The relaxed energy density for isotropic elastic membranes. IMA J. Appl. Math. 36, 85-99 (1986)

12. Steigmann, D.J.: Tension field theory. Proc. R. Soc. Lond. A 429, 141-173 (1999)

13. Chiu, H.C., Benson, R.C., Fiscella, M.D., Burns, S.J.: Mechanical and thermal wrinkling of polymer membranes. J. Appl. Mech. 61, 67-70 (1994)

14. Coman, C.D., Haughton, D.M.: Localized wrinkling instabilities in radially stretched annular thin films. Acta Mech. 185, 179-200 (2006)

15. Coman, C.D.: On the applicability of tension field theory to a wrinkling instability problem. Acta Mech. 190, 57-72 (2007)

16. Plaut, R.H.: Linearly elastic annular and circular membranes under radial, transverse, and torsional loading. Part I: large unwrinkled axisymmetric deformations. Acta Mech. 202, 79-99 (2009)

17. Haughton, D.M., McKay, B.A.: Wrinkling of annular discs subjected to radial displacements. Int. J. Eng. Sci. 33, 335-350 (1995)

18. Haughton, D.M.: Elastic membranes. In: Fu, Y.B., Ogden, R.W. (eds.) Nonlinear Elasticity: Theory and Applications., pp. 233-267. Cambridge University Press, Cambridge (2001)

19. Hornig, J., Schoop, H.: Wrinkling analysis of membranes with elastic-plastic material behavior. Comput. Mech. 35, 153-160 (2005)

20. Danielson, D.A., Natarajan, S.: Tension field theory and the stress in stretched skin. J. Biomech. 135-142 (1975)

21. Mori, D., David, G., Humphrey, J.D., Moore, J.E.: Stress distribution in a circular membrane with a central fixation. J. Biomech. Eng. 127, 549-553 (2005)

22. Massabò, R., Gambarotta, L.: Wrinkling of plane isotropic biological membranes. J. Appl. Mech. 74, 550-559 (2007)

23. Lubarda, V.A., Marzani, A.: Viscoelastic response of thin membranes with application to red blood cells. Acta Mech. 202, 1-16 (2009)—with Addendum, Acta Mech. 212, 215-217 (2010)

24. Lubarda, V.A.: Constitutive analysis of thin biological membranes with application to radial stretching of a hollow circular membrane. J. Mech. Phys. Solids 58, 860-873 (2010)

25. Lubarda, V.A.: Rate theory of elasticity and viscoelasticity for an erythrocyte membrane. J. Mech. Mater. Struct. (2010) (in press)

26. Cerda, E.: Mechanics of scars. J. Biomech. 38, 1598-1603 (2005)

27. Bernal, R., Tassius, C., Melo, F., Géminard, J.-C.: Mechanical characterization of elastic membranes: cell mechanics applications. Appl. Phys. Lett. 90, 063903:1-3 (2007) 
28. Berk, D.A., Hochmuth, R.M., Waugh, R.E.: Viscoelastic properties and rheology. In: Agre, P., Parker, J.C. (eds.) Red Blood Cell Membranes: Structure, Function, Clinical Implications, Hematology Vol 11, pp. 423-454. Marcel Dekker, Inc., New York (1989)

29. Li, X., Steigmann, D.J.: Finite plane twist of an annular membrane. Q. J. Mech. Appl. Math. 46, 601-625 (1993)

30. Haseganu, E.M., Steigmann, D.J.: Analysis of partly wrinkled membranes by the method of dynamic relaxation. Comput. Mech. 14, 596-614 (1994)

31. Steigmann, D.J.: Fluid films with curvature elasticity. Arch. Ration. Mech. Anal. 150, 127-152 (1999)

32. Steigmann, D.J.: Puncturing of thin elastic sheet. Int. J. Non Linear Mech. 40, 255-270 (2005)

33. Evans, E.A., Skalak, R.: Mechanics and Thermodynamics of Biomembranes. CRC Press, Boca Raton (1980)

34. Fung, Y.C.: Biomechanics: Mechanical Properties of Living Tissues. Springer, New York (1993)

35. Shames, I.H., Cozzarelli, F.A.: Elastic and Inelastic Stress Analysis. Taylor \& Francis, London (1997)

36. Helfrich, W.: Elastic properties of lipid bilayers: theory and possible experiments. Z. Naturforsch. 28C, 693-703 (1973)

37. Chiu, H.C., Benson, R.C., Fiscella, M.D., Burns, S.J.: Mechanical and thermal wrinkling of polymer membranes. In: Good, J.K. (ed.) Web Handling, AMD—vol. 149, pp. 37-50. ASME, New York (1992) 